\title{
An Expansion Operator for Interactive Evolution
}

\author{
Jeanine Graf and Wolfgang Banzhaf
}

\begin{abstract}
This paper demonstrates how interactive evolution can be applied to the extrapolation and growth of graphical models. A new operator called expansion is introduced, which plays a significant role in interactive evolution. The expansion operator is based on time series analysis of evolutionary processes. We desribe and demonstrate the function of the new operator.
\end{abstract}

Keywords: Expansion, Progress, Growth, Extrapolation

\section{Interactive Artificial Evolution}

The earth and all living things have evolved through a long history of continual, gradual change shaped by directional natural processes consistent with the laws of physics. Natural evolution of species, as well, as growth and development of individual organisms imply change by continuity. We use interactive eolution and time series analysis to demonstrate development in the world of design and architecture. Our basic assymption is that the complex processes of interactive evolution can be sufficiently represented by different continuous transformations changes.

The novel idea in this paper is to provide the scientist and the designer with a new technique not just to mutate and recombine also to developed and extrapolate models, through the growth and prediction process.

In interactive evolution (IE), the user selects one or more individual(s) which survive(s) and reproduce(s) (with variation) to constitute a new generation. These techniques can be applied to the production of computer graphics, creating forms. Potential applications of interactive evolution include artificial life design, e.g., development of components of biological nature and engineering construction design. The best-known representatives of this class of algorithms are evolutionary programming (EP), developed in the U.S. by L.J. Fogel (Fogel, et al. 1966). evolution strategies (ESs), developed in Germany by I. Rechenberg (1973) and H.-P. Schwefel (1981), and genetic algorithms (GAs), developed in the U.S. by J.H. Holland.

Due to the nature of the applied operators in an interactive evolution, mostly intermediate models will result as variants. In order not to converge too quickly to suboptimal solutions, a mechanism should be provided by which evolution can leave the model area already present. For this purpose, we define a new genetic operator called expansion, which play significant role in interactive evolution. Each iteration of the IE comprises four phases: recombination, mutation, expansion, and reproduction. A top-level description is as follows: 
Step 1: Start with an initial time $\mathrm{T}:=0$;

Initialize an initial population $\mathrm{P}(0)$ of graphic models $\mathrm{M}$.

Compute the fitness function $\mathrm{f}(\mathrm{mi})$ for each object $\mathrm{mi}$.

Step 2: Apply the selection with the interactive system by picking the best parents for offspring production.

Step 3: Select pairs of objects and apply recombination and/or mutation and/or expansion. Create new generation of models by mating current models; increase the time counter; set $\mathrm{T}:=\mathrm{T}+1$

Step 4: Compute the fitness function $\mathrm{f}\left(\mathrm{m}^{\prime} \mathrm{i}\right)$ for each object $\mathrm{m}^{\prime} \mathrm{i}$.

step 5: increase the time counter; set $\mathrm{T}:=\mathrm{T}+1$ repeat until you get the best models solution.

\section{Simulated models and the Expansion Operator}

\subsection{Evolving Simulated Models}

In simulated evolution, one approach is to consider an object as a set of points. Each point can be defined using two or three coordinates, $\mathrm{x}, \mathrm{y}$ and $\mathrm{z}$. The process of altering or of growth can be systematically implemented using the concept of transforming it. A transformation is determined by defining new variables, $\mathrm{x}^{\prime}, \mathrm{y}^{\prime}$ and $\mathrm{z}^{\prime}$, as functions of $\mathrm{x}, \mathrm{y}$ and $\mathrm{z}$. A transformation can often be considered as a general way of change, independent of the special object to which is applied. The methods uses corresponding points in the models to be interpolated.

In interactive evolution, techniques which can be used for object metamorphosis also include solid deformations. In such a case, the 2-D or 3-D model coordinates of a object can be considered as a sequence evolution from generation 0 to generation $\mathrm{T}$, which can be extrapolated in order to assume the shape and properties of a new model for a generation $\mathrm{T}+1$.

Any 2-D shape can be represented such as a sequence of points or vertices, with each vertex consisting of an ordered pair of numbers, its coordinates. The complete array of pixel values of a 2-D image, however, has nothing to do with the structure being represented in the model. Thus we use interpolation techniques for the to generate new variants of existing models, because structural or functional conservation of the model content is of most importance in applications.

\subsection{The expansion operator}

Graphical models are to be predicted on condition, that a large body of historical information is avaible on its past evolution. In time series analysis historical data on the variable to be forecast are analysed in an attempt to identify a data pattern. Then, assuming that the pattern will continue in to the future, this pattern is extrapolated in order to produce forecasts. Linear prediction as a extrapolation technique, with a most straightforward use of the coefficients in predicting the future of a time series from a record of its past. Linear prediction is especially successful at extrapolating signals which are smooth and oscillatory, though not necessarily periodic.

A short description of the expansion operator is the following: Let $t \in \mathbf{N}$ be the generation counter, with $t=0$ initial population, $t=T$ the present generation, then the sequence $\mathbf{H}:=\left(\mathbf{M}_{i}^{0}, \ldots, \mathbf{M}_{i}^{t}, \ldots, \mathbf{M}_{i}{ }^{T}\right)$ constitutes a time series of models specified in terms of components and their coordinates. This time series can be used to generate a new expanded model $\mathbf{M}_{i}{ }^{T+1}$ which is a prediction or extrapolation of the evolutionary process already going on from $t=0$ to $t=T$. Thus the expansion operator provides predictive models of the evolutionary specifically, process we use autoregressive models as a particular case of linear regression models.

To understand the method of extrapolation of deformed models, it is useful to introduce some formalism. Suppose we have a time series of $T$ observations, denoted by $f_{1}, f_{2}, \ldots, f_{T}$. These are regarded as observed values of the random variables $F_{1}, F_{2}, . ., F_{T}$. For every point in a shape of a model, we have a variable $F$. We wish to forecast the value of the time series $L$ time periods into the future. This value is denoted 
by $f_{T+L}$ and is one instantiation of the random variable $F_{T+L}$. Of course, $f_{T+L}$ is currently unknown, but a forecast of $f_{t+L}$, based on the observed values $f_{1}, f_{2}, \ldots, f_{T}$ can be derived and is denoted by $\hat{f}_{T}(L)$. This forecast is said to have origin $T$ and lead time $\mathrm{L}$.

By asserting that the noticed expansion operator is stationary, we are requiring that the underlying variables $F_{1}, F_{2}, \ldots$ have the same mean $\mu$ and the same variance $\sigma_{f}{ }^{2}$. The simplest possible model for a stationary expansion operator is one in which the variables $F_{t}$ are independent and have the same normal probability distribution for each $t$. To express the properties of the variable $F_{t}$, we use the notation $F_{t} \sim N\left(\mu, \sigma_{f}^{2}\right)$. We then introduce a sequence of variables $A_{t}$ as follows; $F_{t}=\Theta_{0}+A_{t}$, where $A_{t} \sim N\left(0, \sigma_{A}{ }^{2}\right)$. The autoregressive model new assumes that $F_{t}$ does only depend on the variable $F_{t-1}$, $F_{t-2}, \ldots$, immediately preceding it. More precisely, we will assume that the variable $F_{t}$ is a linear function of the two preceding variables $F_{t-1}$ and $F_{t-2}$, plus a random shock $A_{t}$. This gives the model

$$
\begin{aligned}
& F_{t}=\Theta_{0}+\phi_{1} F_{t-1}+\phi_{2} F_{t-2}+A_{t} \\
& \text { where } A_{t} \sim N\left(0, \sigma_{A}^{2}\right)
\end{aligned}
$$

This is called an autoregressive model of order 2, or, more briefly, an AR2 model with coefficients $\phi_{1}, \phi_{2}$. The parameters $\phi_{1}$ and $\phi_{2}$ must satisfy a number of conditions for this model to be a stationary stochastic process. These conditions for are :

$\phi_{1}+\phi_{2}<1$

$\phi_{1}-\phi_{2}<1$,

$-1<\phi_{2}<1$.

It is advisable at this point to notice that the concept of a time series model, assumes an underlying stochastic process. We cannot observe this underlying stochastic process and so we can never be absolutely sure what we have correctly identified it. The most that we can say is that we have found a model, a realisation which characteristic properties similar to those of the observed time series.

\subsection{Application of the Extrapolation and Prediction}

\section{Phenotypes and Genotypes}

In interactive evolution of graphical models, genotypes are represented as numerical data, real values which are code for points, vertices and shapes. The phenotype is the product of an interpretation of the underlying genotypic representation. In our case, the phenotype is the resulting graphical model on the screen.

The genotype of the new model are coordinates, which are predicted through abovementioned time series analysis. Through the interpolation of these coordinates a new model can be generated and displayed as a new phenotype.

To extrapolate the models, we must first compute new locations for all points in the genotype such that points at the tail of a displacement vector are moved to the respective target position at the head of the vector, which we define with help of time series analysis or prediction. All other points are moved along in a consistent manner such that neighbour relations between point remain unchanged whenever possible.

In the following, we will present an example of a new model in generated by extrapolation of a sequence of models, through the expansion operator.

Every genotypic point gets an equation of extrapolation which is used to predict the new point in a descendants, see Table.

\section{Expansion Operator in JARDIN System}

Jardin is a computer program written to be able to evolve any graphical model with interactive evolution. It runs under $X$ Windows. For the expansion operator, the user just needs to give a sequence of graphical models as input, with some points characteristic of the shape of the images. As a result of the operator 
action, the user gets new models by way of prediction from the past.

We demonstrate how the expansion operator in interactive evolution can be applied to graphical models, and how we can predict or extrapolate new models with time series analysis. The graphical models are based on a physical models with each of which constructed from shapes and points. A solution can be found whereby the series of shapes or points is streched into a new model with a minimum amount of work required by the user.

These techniques can be applied to the design of cars, airplanes, engineering components and construction projects. The expansion operator has shown that interesting results can be achieved even with a small sequence of models to be predicted from. This might make the system applicable to problems of ontogeny, development of design models or growth. One of the main benefits from this technique is the ability to help the human user to get neew ideas and to increase her / his creativity.

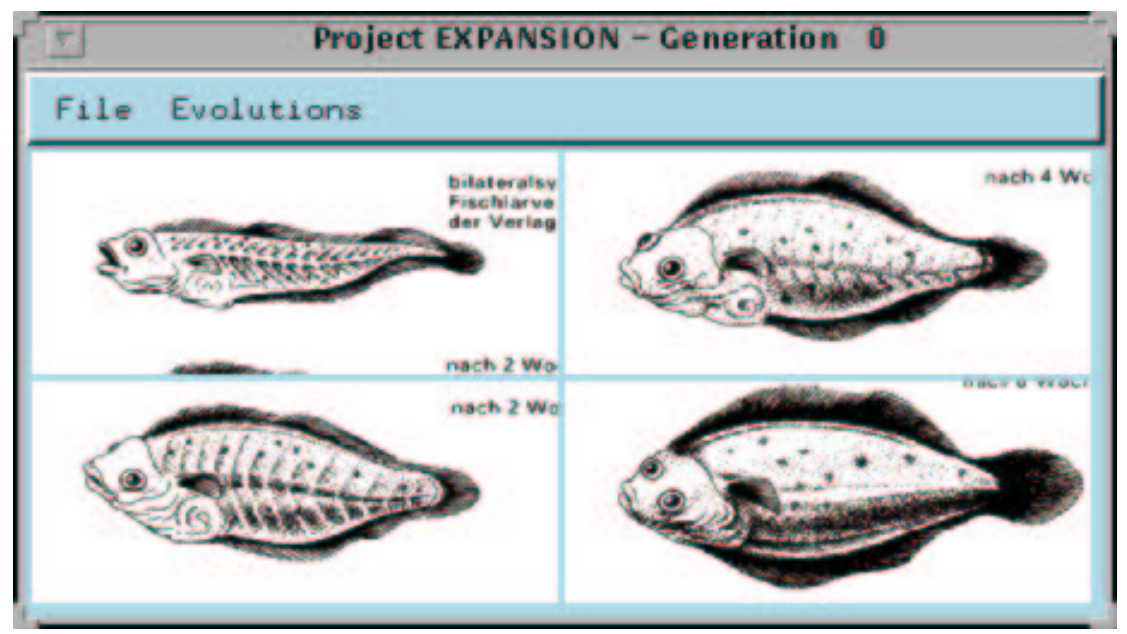

Figure 1: A sample sequence of four fish models taken from actual biological ontogeny with time elapsed between images in the order of some weeks.

This table shows some values of four fish models, and new coordinates are produced through time series analysis, these coordinates belong two a shape area in the models, which produce new models.

\begin{tabular}{|c|c|c|c|c|c|}
\hline No. & Model1 & Model2 & Model3 & Model4 & pre \\
\hline$\overline{1}$ & x: 25 y: 44 & x: 24 y: 42 & x: 23 y: 52 & x: 25 y: 44 & "x: 22.6 y: 56.4 \\
\hline 2 & $x: 34 y: 32$ & $: 34 \mathrm{y}: 33$ & $x: 34$ y: 37 & $x: 34$ y: 33 & $\mathrm{x:} 34.0 \mathrm{y}: 39.3$ \\
\hline 3 & $x: 62$ y: 31 & $x: 61$ y: 23 & $x: 63$ y: 29 & $x: 61$ y: 23 & $\mathrm{x}: 63.6 \mathrm{y}: 27.4$ \\
\hline 4 & $x: 97 y: 34$ & $x: 95$ y: 21 & $x: 95 y: 22$ & $\mathrm{x}: 97 \mathrm{y}: 18$ & x: 100.8 y: 16.3 \\
\hline 5 & $\mathrm{x}: 129$ y: 39 & $x: 132 y: 34$ & $\mathrm{x}: 136 \mathrm{y}: 35$ & $x: 135$ y: 24 & $\mathrm{x}: 141.4 \mathrm{y}: 30.2$ \\
\hline 6 & $\mathrm{x}: 150 \mathrm{y}: 39$ & $\mathrm{x}: 159 \mathrm{y}: 44$ & $\mathrm{x}: 160 \mathrm{y}: 41$ & $x: 168 \mathrm{y}: 35$ & $\mathrm{x}: 170.4 \mathrm{y}: 28.2$ \\
\hline 7 & $\mathrm{x}: 168 \mathrm{y}: 44$ & $x: 169 y: 52$ & $\mathrm{x}: 168 \mathrm{y}: 48$ & $\mathrm{x}: 179 \mathrm{y}: 49$ & $\mathrm{x}: 173.37 \mathrm{y}: 48.1$ \\
\hline 8 & $\mathrm{x}: 136 \mathrm{y}: 53$ & $x: 135 y: 59$ & $x: 138$ y: 62 & $\mathrm{x}: 138 \mathrm{y}: 67$ & x: 140.8 y: 71.4 \\
\hline$\overline{9}$ & $x: 91$ y: 59 & $x: 98$ y: 65 & $x: 84$ y: 70 & $\mathrm{x}: 85 \mathrm{y}: 75$ & $x: 75.8$ y: 80.3 \\
\hline$\overline{10}$ & $x: 53$ y: 59 & $x: 49$ y: 60 & $x: 52$ y: 68 & $x: 52$ y: 68 & $\mathrm{x}: 53.6 \mathrm{y}: 76.8$ \\
\hline$\overline{11}$ & $x: 43$ y: 38 & $x: 44$ y: 39 & $x: 43$ y: 48 & $x: 43$ y: 48 & $x: 42.6$ y: 58.1 \\
\hline
\end{tabular}




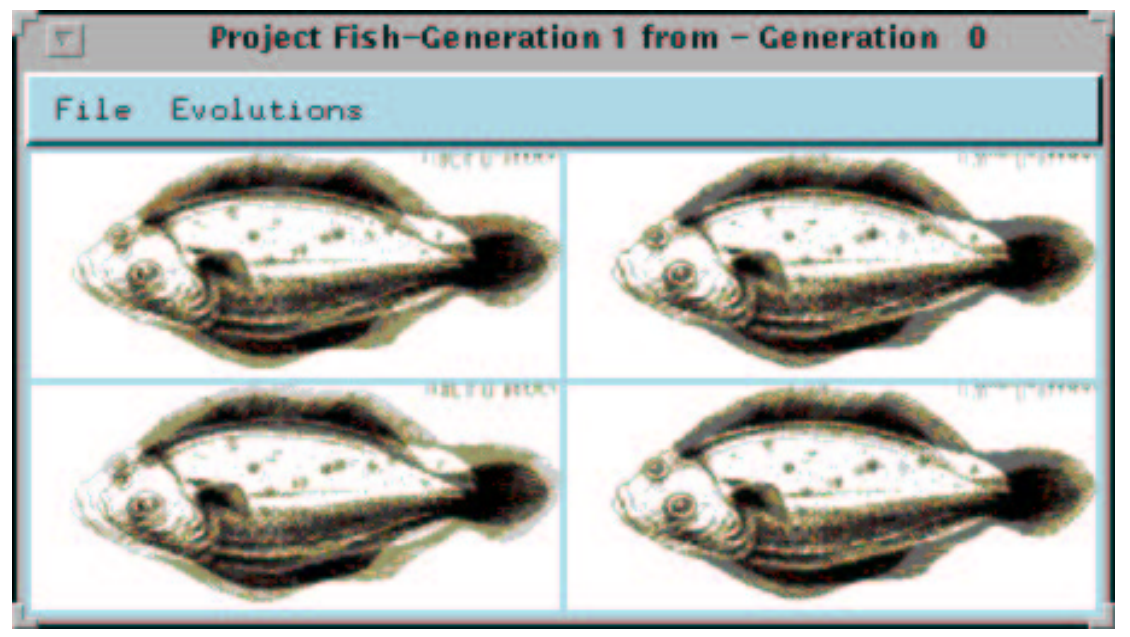

Figure 2: An example of the result of applying the expansion operator to the sequence of Figure 1. Models are predicted using autoregressive modeling of second order based on 11 points in a time series analysis.

\section{References}

L. J. Fogel, A. J. Owens, and M. J. Walsh. Artificial Intelligence through Simulated Evolution. Wiley, New York, 1966.

J. H. Holland. Adaption in Natural and Artificial Systems. Ann Arbor, The University of Michigan Press, 1975.

J. Graf, W. Banzhaf. Interactive Evolution of Images. EP95, In print, 1995.

I. Rechenberg. Evolutionsstrategie: Optimierung technischer Systeme nach Prinzipien der biologischen Evolution. Frommann-Holzboog, Stuttgart, 1973.

H.-P. Schwefel. Numerical Optimization of Computer Models. Wiley, NY, 1981.

P. Young Recursive Estimation and Time-Series Analysis. Springer-Verlag, NY, 1984. 


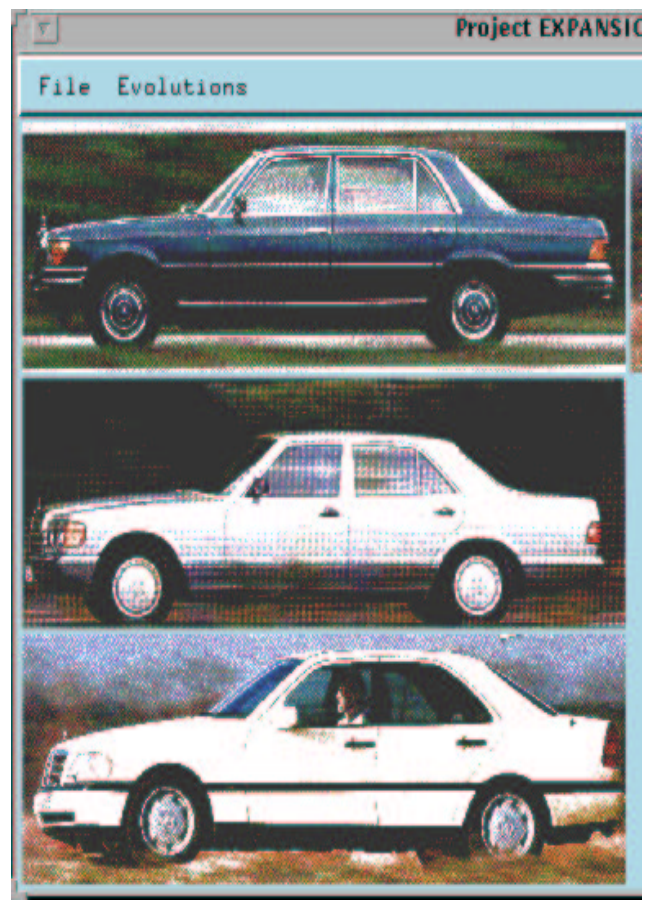

Figure 3: Three more recent car models of a major German manufacturer, taken as design development.

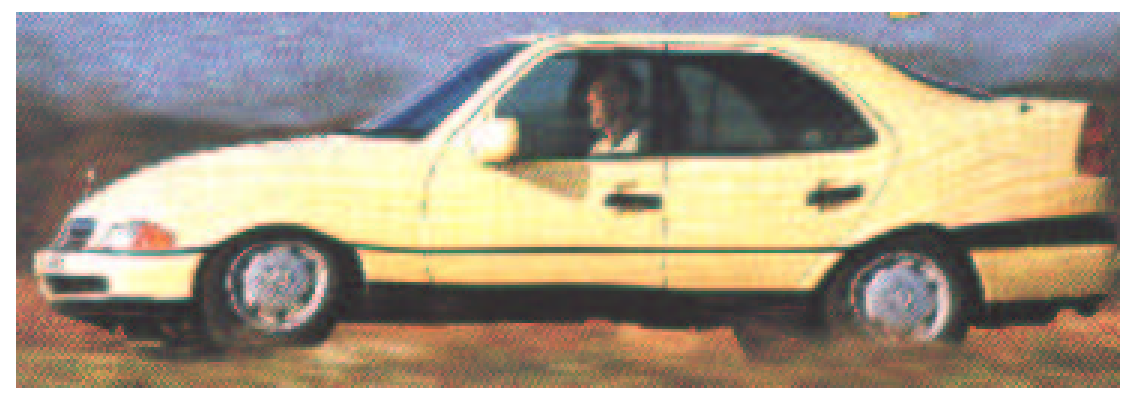

Figure 4: Predicted shape of a future car model based on the results of the time series analysis. Only to be taken as indicating a tendency possibly inspiring a designer 